\section{A Method for Quantitative Starch-gel Electrophoresis}

EFForts to make use of starch-gel electrophoresis oven in quantitative protein analysis has led to several techniques to mako the gel transparent ${ }^{1-5}$. A further method, where the gel is stained in the same modium which holps it become transparent, is described hero.

The gol is cut with a device giving a slice $1 \mathrm{~mm}$. thick with even surfaces on both sides. The slice is immersedin a $95^{\circ} \mathrm{C}$. bath of $47 \cdot 5: 47 \cdot 5: 5(\mathrm{v} / \mathrm{v} / \mathrm{v})$ glycerol water/acetic acid saturated with amido black $10 B$ for $10 \mathrm{~min}$. and then washed at room temperaturo with slow, continuous shaking in a series of glycerol/ water mixtures where the glycerol content is increasing, giving a final wash medium of pure glycerol. The washing proceduro is somewhat slow, taking two days. The slice can then be analysed in a chromatograph for transmitted light using a supporting device. The data of two normal human scra and two myeloma sera analysed using an electrophoresis method enabling the separation of 14 fractions and a transmission chromatograph (Spinco 'Analytrol') are presented in Tablo 1. Paper eloctrophoresis data of the same normal sera are presented in Table 2 . For comparison the data of some normal sera analysed using the same electrophorosis method as in Table 1, but the original methanol-staining method and a reflexion chromatograph (Joyce, Loewoll and Co. 'Chromograph') are presented in Table 3. The striking difference in the amount of albumin fraction obtained by the two methods emphasizes the need for different normal values when using different methods.

EVA VAHVASELKä

Stato Serum Instituto, Helsinki.

1 Smithies, O., Adv. Prot, Chem., 14, 65 (1959).

"Fine, J. M., et al., Nature, 181, 1152 (1958).

${ }^{3}$ Habermann, E., and Szopa, B., Z. ges. exp. Med., 131, 520 (1959).

Rubinstein, H. J., et al., Clin. Chim. A eta, 5, 583 (1960).

"Johns, E. W., J. Chromatog., 5. 91 (1961).

\section{Structural Revisions in the $\omega$-Halobenz- aldehyde Arylhydrazone Series}

Some twenty compounds, described as 3-keto-1,2endo-arylimino-1,2-dihydro-1,2-benzisodiazole 1-oxides (I), have beon prepared by Chattaway et al..$^{1-8}$; eleven of these havo been reduced by stannous chloride in hydrochloric acid (or, in some cascs, by prolonged boiling in ethanol) to deoxy compounds, formulated as (II $)^{2-6}$.

A recent study of the Chattaway-Adamson rearrangement $t^{4,0}$ has directod attention to these interesting compounds. 'The 'oxides' result from mild basic treatment of the appropriate $\omega$-halo-o-nitrobenzaldehydo arylhydrazones; from a mechanistic point of view ${ }^{10}$, these compounds should be regarded as 3-arylazo-anthranil 1-oxides (III), and, as such, are secn to bo analogous to tho isoxazoline oxides of Kohler ${ }^{11}$. Such a conclusion is supported by the electronic and vibrational spectra of compound (III, $A r=2,4$-dibromophenyl), and is in harmony with the

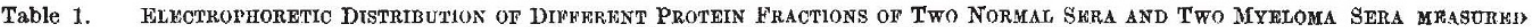

\begin{tabular}{|c|c|c|}
\hline Fraction & Run 1 & $\begin{array}{l}\text { Normal serum } 1 \\
\text { Run } 2\end{array}$ \\
\hline $\begin{array}{l}\text { Pre-albumin } 1 \\
\text { Pre-albumin } 2 \\
\text { Albumin } \\
\text { Post-albumin } 1 \\
\text { Post-albumin } z \\
\text { Corruloplasmin } \\
\text { Transferrin } \\
\alpha_{1}-\beta-\text {-Globulins }\end{array}$ & $\begin{array}{r}1 \cdot 7 \\
1 \cdot 8 \\
57 \cdot 0 \\
1 \cdot 9 \\
4 \cdot 2 \\
4 \cdot 4 \\
1 \cdot 9\end{array}$ & $\begin{array}{r}1 \cdot 5 \\
1 \cdot 6 \\
56 \cdot 5 \\
2 \cdot 4 \\
4 \cdot 8 \\
3 \cdot 2 \\
2 \cdot 4\end{array}$ \\
\hline Haptoglobins & $5 \cdot 2$ & $\{4 \cdot 0$ \\
\hline $\begin{array}{l}\text { 'Slow' } a_{\mathbf{a}} \text {-globulin } \\
\beta \text {-Lipoprotein }\end{array}$ & $\begin{array}{l}2 \cdot 6 \\
6 \cdot 1\end{array}$ & $\begin{array}{r}4.0 \\
6.8\end{array}$ \\
\hline $\begin{array}{l}\gamma \text {-Globulin } \\
\text { Myelomaprotein }\end{array}$ & $\begin{array}{r}13 \cdot 2 \\
\ldots\end{array}$ & $12 \cdot 8$ \\
\hline
\end{tabular}

Table 2. EIACCIROPHORETIC DISTRIBdTION OF DIFFERENT PROTEIN FRAOTIONS OF THE SAME TWO NORMAL SERA $\triangle S$ IN TABLE 1 MEAS-
URED BY PAPER ELECTROPHORLSIS

Fraction

\begin{tabular}{lrr}
\multicolumn{1}{c}{ Fraction } & Normal serum 1 & Normal serum 2 \\
Albumin & $58 \cdot 3$ & $64 \cdot 5$ \\
$\alpha_{1}$-Globulin & $4 \cdot 9$ & $3 \cdot 9$ \\
$\alpha_{2}$-Globulin & $8 \cdot 7$ & $5 \cdot 2$ \\
$\beta$-Globulin & $10 \cdot 6$ & $10 \cdot 3$ \\
$\gamma$-Globulin & $17 \cdot 5$ & $16 \cdot 2$
\end{tabular}

Table 3. Fuedrophoretic Distribetion of Different Protein FRACTIONS OF FOUR NORMAL SERA MMASURED BY STARCH-GEL FRACTIONS OF FOUR NORAL SERA MRASURET BY

\begin{tabular}{|c|c|c|c|c|}
\hline Fraction & 1 & 2 & 3 & 4 \\
\hline $\begin{array}{l}\text { Pre-albumin } 1 \\
\text { Pre-glbumin } 2\end{array}$ & $\begin{array}{l}1 \cdot 3 \\
4 \cdot 6\end{array}$ & $1 \cdot 6$ & $\begin{array}{l}2 \cdot 8 \\
3 \cdot 4\end{array}$ & $\begin{array}{l}1 \cdot 3 \\
2 \cdot 3\end{array}$ \\
\hline Albumln & $33 \cdot 1$ & $36 \cdot 0$ & $37 \cdot 3$ & $39 \cdot 3$ \\
\hline Post-albumin 1 & $2 \cdot 2$ & $2 \cdot 3$ & $2 \cdot 2$ & $2 \cdot 0$ \\
\hline Post-albumin 2 & $2 \cdot 9$ & $2 \cdot 7$ & $\overline{3} \cdot \overline{5}$ & $2 \cdot 1$ \\
\hline Coruloplasmin & 8.7 & $4 \cdot 9$ & $12 \cdot 2$ & $5 \cdot 2$ \\
\hline Transferrin & $4 \cdot 3$ & $7 \cdot 7$ & $5 \cdot 2$ & $6 \cdot 8$ \\
\hline$\alpha_{1}-\beta$-Clobulins & $4 \cdot 5$ & 3.8 & $3 \cdot 2$ & $3 \cdot 8$ \\
\hline Haptoglobins & $4 \cdot 5$ & $3 \cdot 1$ & $3 \cdot 2$ & $3 \cdot 6$ \\
\hline 'Slow' $a_{\mathbf{a}}$-Globulin & $11 \cdot 2$ & $7 \cdot 7$ & $6 \cdot 4$ & $6 \cdot 9$ \\
\hline$\beta$-Lipoprotein & $3 \cdot 4$ & 8.5 & $4 \cdot 5$ & $7 \cdot 0$ \\
\hline$\gamma$-Globulin & $13 \cdot 2$ & $14 \cdot 7$ & $13 \cdot 5$ & $16 \cdot 9$ \\
\hline
\end{tabular}

Run 1
$1 \cdot 5$
$1 \cdot 6$
$64 \cdot 8$
$1 \cdot 2$
$1 \cdot 9$
$3 \cdot 2$
$0 \cdot 8$
$1 \cdot 6$
$0 \cdot 8$
$3 \cdot 5$
$2 \cdot 9$
$16 \cdot 2$
-

Normal serum 2
Run 2
$1 \cdot 0$
$1 \cdot 4$
$65 \cdot 0$
$1 \cdot 5$
$1 \cdot 0$
$1 \cdot 5$
$3 \cdot 4$
$1 \cdot 5$
$2 \cdot 9$
$2 \cdot 4$
$1 \cdot 9$
$14 \cdot 5$
$\cdots$

Run 3
$1 \cdot 1$
1.3
$B 1.2$
1.6
1.0
1.6
3.2
1.0
1.6
1.1
1.1
4.8
3.2
16.2
-

$\begin{array}{cc}\text { Mycloma } & \text { Myeloma } \\ 1 & 2 \\ 1 \cdot 6 & 1 \cdot 5 \\ 1 \cdot 1 & 1 \cdot 0 \\ 43 \cdot 4 & 45 \cdot 6 \\ 1 \cdot 0 & \{2 \cdot 0 \\ 1 \cdot 5 & 4 \cdot 1 \\ 4 \cdot 8 & 6 \cdot 1 \\ 4 \cdot 2 & 6 \cdot 1 \\ 4 \cdot 6 & 3 \cdot 1 \\ 2 \cdot 6 & 2 \cdot 0 \\ 3 \cdot 7 & 1 \cdot 0 \\ 29 \cdot 2 & 5 \cdot 1 \\ 8 \cdot 9 & 5 \cdot 6 \\ - & 8 \cdot 2\end{array}$

intense colour and with the chemical properties of these labilo compounds.

Reduction to the near-colourless deoxy-compounds evidently involves scission of tho heterocyclic ring, followed by recyclization to the mesoionic indazole, derivatives (TV). This is implied by the genoral

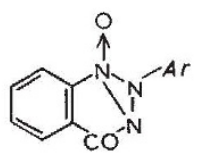

(I)<smiles>COc1ccccc1N1CCCC1</smiles>

(II)

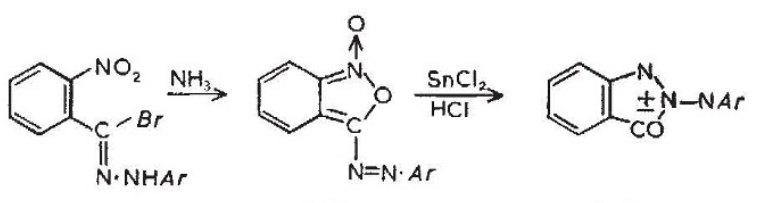

(1II)
(IV) 